\title{
Determination of each neutral oligosaccharide in the milk of Japanese women during the course of lactation
}

\author{
Wataru Sumiyoshi ${ }^{1,2}$, Tadasu Urashima ${ }^{1}$, Tadashi Nakamura ${ }^{1}$, Ikichi Arai ${ }^{1}$, Tadao Saito ${ }^{3}$, \\ Norihiko Tsumura $^{4}$, Bing Wang ${ }^{5}$, Janette Brand-Miller ${ }^{5}$, Yoko Watanabe ${ }^{6}$ and Kazumasa Kimura ${ }^{6}$ \\ ${ }^{1}$ Department of Bioresource Science, Obihiro University of Agriculture and Veterinary Medicine, Inada cho, Obihiro, \\ Hokkaido, 080-8555, Japan \\ ${ }^{2}$ Course of the Science of Bioresources, The United Graduate School of Agricultural Science, Iwate University, \\ 18-8 Ueda 3-chome, Morioka, Iwate, 020-8550, Japan \\ ${ }^{3}$ Department of Bioproduction, Graduate School of Agriculture, Tohoku University, Tsutsumidori-Amamiya machi 1-1, \\ Aoba-ku, Sendai, Miyagi, 981-8555, Japan \\ ${ }^{4}$ Department of Obstetrics and Gynecology, Obihiro Kosei General Hospital, Obihiro, Hokkaido, 080-0016, Japan \\ ${ }^{5}$ Human Nutrition Unit, Department of Biochemistry, University of Sydney, NSW, 2006, Australia \\ ${ }^{6}$ YAKULT Central Institute for Microbiological Research, 1796 Yaho, Kunitachi, Tokyo, 186-8650, Japan
}

(Received 12 July 2001 - Revised 11 February 2002 - Accepted 9 September 2002)

\begin{abstract}
Using reverse-phase HPLC after pyridylamination, we quantified the concentrations of major neutral oligosaccharides in the milk of sixteen Japanese women collected at 4, 10, 30 and $100 \mathrm{~d}$ postpartum. In colostrum and mature milk ( $30 \mathrm{~d}$ lactation), lacto-N-fucopentaose (LNFP) I was the most abundant oligosaccharide, followed by $2^{\prime}$-fucosyllactose $\left(2^{\prime}\right.$-FL $)+$ lacto-N-difucotetraose (LNDFT), LNFP II + lacto-N-difucohexaose II (LNDFH II), and 3-fucosyllactose (3-FL). Together these accounted for $73 \%$ of the total weight of neutral oligosaccharides in colostrum and mature milk. Changes in concentration occurred during the course of lactation. LNFP I and 2'-FL + LNDFT increased from 4 to $10 \mathrm{~d}$ postpartum, and then declined by $100 \mathrm{~d}$. LNFP II + LNDFH II steadily increased during the first $30 \mathrm{~d}$ and then declined. In contrast, 3-FL increased steadily throughout the entire $100 \mathrm{~d}$ of study. Large differences were observed between our data and previously published data in Italian women, in terms of both the concentration and temporal changes of each oligosaccharide. These differences may be caused by different assay methodology, although racial differences cannot be ruled out.
\end{abstract}

Human milk oligosaccharides: Lactation period: Pyridylamination: High-performance liquid chromatography

The oligosaccharide fraction of human milk is the third largest solid component following lactose and lipid (Newburg \& Neubauer 1995). Mature human milk contains $12-14 \mathrm{~g}$ oligosaccharides/l while human colostrum contains 20-24 g/l. Using matrix-assisted laser desorption/ionization MS (Stahl et al. 1994) and other methods, approximately 130 oligosaccharides have been found in human milk, with about eighty of them characterized. Based on their core structure, they are classified in twelve families. The secretor and Lewis phenotypes of lactating woman determine the structure of the terminal group of these carbohydrate chains, and have been used to characterize the
Lewis a and Lewis b blood group determinants (Watkins, 1966; Thurl et al. 1997).

Breast-fed infants appear to have fewer or less severe gastrointestinal and respiratory infections during the first year of life than formula-fed infants. Milk oligosaccharides may play a protective role. In the first instance, they act as a growth factor for intestinal bacteria such as Bifidobacterium bifidum (Gÿorgy et al. 1954; Yoshioka et al. 1983; McVeagh \& Miller, 1997; Newburg, 2000). Human milk oligosaccharides containing $\mathrm{N}$-acetylglucosamine are essential for the growth of B. bifidum (Gÿorgy \& Rose, 1955). Bifidobacteria produce lactic acid by lactose fermentation,

\footnotetext{
Abbreviations: 2'-FL, 2'-fucosyllactose; 3-FL, 3-fucosyllactose; LNDFH, lacto-N-difucohexaose; LNDFT, lacto-N-difucotetraose; LNFP, lacto-Nfucopentaose; LNH, lacto-N-hexaose; LNnH, lacto-N-neohexaose; LNnT, lacto-N-neotetraose; LNT, lacto-N-tetraose.

* Corresponding author: Dr T. Urashima, fax +81 15549 5577, email urashima@obihiro.ac.jp
} 
increasing the acidity of the intestinal environment, and thereby inhibiting the growth of pathogenic organisms.

Second, milk oligosaccharides act as direct inhibitors to pathogenic organisms (Andersson et al. 1986; Coppa et al. 1990, 2000; Dai et al. 1990; Cravioto et al. 1991; Kunz \& Rudloff, 1993; Cervantes et al. 1995; Idota et al. 1995; Newburg, 1996, 1997, 1999; Zopf \& Roth, 1996; McVeagh \& Miller, 1997; Portelli et al. 1998; Miller \& McVeagh, 1999; Newburg et al. 1999). It is known that some human milk oligosaccharides have structures analogous to glycoproteins and glycolipids on cell surface receptors for pathogens and that they bind competitively to pathogens in the gastrointestinal, respiratory, and urinary tract.

The amounts of oligosaccharides increase or decrease in human milk during the course of lactation to protect infants from viral or bacterial attack, but quantitative data for individual oligosaccharides are scarce (Coppa et al. 1999; Nakhla et al. 1999; Erney et al. 2000; Chaturvedi et al. 2001). Coppa et al. (1999) reported changes in several oligosaccharides in the milk of Italian women at different stages of lactation, but it is not known if this is representative of all women.

In the present study we report quantitative data on several neutral oligosaccharides in the milk of Japanese women throughout the course of lactation. In some quantitative methods of sugars utilizing pre-column derivatization (Chaturvedi et al. 1997; Yasuno et al. 1999), we used reductive amination with 2-aminopyridine and subsequent HPLC to determine each oligosaccharide content in the milk, because the procedures are easy and the derivatives are very sensitive to being quantified in HPLC analysis. These are well-established methods for the analysis and determination of some oligosaccharides and carbohydrate moieties of glycoproteins (Hase, 1994; Saito et al. 1998; Yanagida et al. 1999). The pyridylamination reaction proceeds in two steps; a coupling reaction (Schiff base formation) and reduction of the Schiff base. It has been reported that the methods have high recovery and reproducibility $( \pm 2 \%)$ and allow the determination of picomole levels of neutral and amino sugars (Takemoto et al. 1985). We used only a small quantity of milk $(1 \mathrm{ml})$ to determine each oligosaccharide content with this method.

\section{Materials and methods}

\section{Materials}

Human milk samples were collected from sixteen women at $4,10,30$ and $100 \mathrm{~d}$ postpartum. Two samples, one collected at $10 \mathrm{~d}$ and one at $100 \mathrm{~d}$ postpartum, were excluded from the analysis. All samples (approximately $3-5 \mathrm{ml}$ ) at each lactation period were collected by hand into sterilized tubes, which were then stored at $-40^{\circ} \mathrm{C}$ until use. The $\mathrm{ABO}$ blood group of the donors was determined: $\mathrm{A}$, three; $\mathrm{B}$, five; O, seven; $\mathrm{AB}$, one. The Rhesus blood group of all donors was positive.

3-Fucosyllactose (3-FL) and lacto-N-fucopentaose (LNFP) III were purchased from Funakoshi Co., Tokyo, Japan, and $2^{\prime}$-fucosyllactose (2'-FL), lacto-N-difucohexaose (LNDFH) II and lacto-N-hexaose (LNH) were from Seikagaku Co., Tokyo, Japan. Lacto-N-tetraose (LNT),
lacto-N-neotetraose (LNnT), lacto-N-difucotetraose (LNDFT), LNFP I, LNFP II, LNDFH I and lacto-N-neohexaose $(\mathrm{LNnH})$ were obtained from Sigma, St Louis, MO.

\section{Preparation of each oligosaccharide fraction from human milk}

The milk samples were thawed and a portion of each $(1 \mathrm{ml})$ was extracted with 4 volumes of chloroform-methanol $(2: 1, \mathrm{v} / \mathrm{v})$. The emulsion was centrifuged at $3500 \mathrm{rpm}$ for $30 \mathrm{~min}$ at $4^{\circ} \mathrm{C}$ and the lower chloroform layer and denatured protein were discarded. The upper layer was collected and the fraction was evaporated and then freezedried. The resulting powders were called the 'carbohydrate fraction'.

The carbohydrate fraction was dissolved in water $(1 \mathrm{ml})$ and the solution passed through a Bio Gel P-2 (Extra fine, $<45 \mu \mathrm{m}$; Bio Rad Laboratories, USA) column $(2.6 \times$ $100 \mathrm{~cm}$ ) which had been calibrated with $2 \mathrm{mg}$ of each of galactose (monosaccharide), lactose (disaccharide), raffinose (tri-saccharide). Elution was done with water at a flow rate of $15 \mathrm{ml} / \mathrm{h}$ and ninety-five fractions of $5 \mathrm{ml}$ were collected. Aliquots $(0.25 \mathrm{ml})$ of each fraction were analysed for hexose using the phenol- $\mathrm{H}_{2} \mathrm{SO}_{4}$ method (Dubois et al. 1956) and for sialic acid with the periodate-resorcinol method (Jourdian et al. 1971). Peak fractions were pooled and freeze-dried.

Of fractions separated by the gel chromatography, the fraction, which was positive by the periodate-resorcinol method, was dissolved in $1 \mathrm{ml}$ of $50 \mathrm{~mm}$-Tris-hydroxyaminomethane (Tris)- $\mathrm{HCl}$ buffer ( $\mathrm{pH} 8.7$ ) and subjected to anion exchange chromatography with the DEAE Sephadex A-50 (Amersham Pharmacia Biotech UK Ltd., UK) column $(1.0 \times 40 \mathrm{~cm})$ equilibrated with the same buffer. The unadsorbed components containing the neutral oligosaccharide fraction were eluted with the same buffer $(100 \mathrm{ml})$ and the adsorbed components containing the acidic oligosaccharide fraction were eluted with $300 \mathrm{ml}$ of linear gradient from 0 to $1.0 \mathrm{~mm}-\mathrm{NaCl}$ in $50 \mathrm{~mm}$-Tris$\mathrm{HCl}$ buffer ( $\mathrm{pH} \mathrm{8.7)}$. Elution was done at a flow rate of $15 \mathrm{ml} / \mathrm{h}$ and fractions of $5 \mathrm{ml}$ were collected. Aliquots $(0.25 \mathrm{ml})$ of each fraction were analysed for hexose. Peak fractions were pooled and freeze-dried. The neutral oligosaccharide in the unadsorbed fraction was dissolved in water $(1 \mathrm{ml})$ and passed through a Bio Gel P-4 (Bio Rad, Extra fine, $<45 \mu \mathrm{m})$ column $(2.6 \times 100 \mathrm{~cm})$ under the same condition as in the gel chromatography with Bio Gel P-2. Peak fractions were pooled and freeze-dried.

\section{Quantification of each neutral oligosaccharide}

The pyridylaminated derivative of each neutral oligosaccharide fraction was prepared according to the method of Kondo et al. (Kondo et al. 1990; Suzuki et al. 1991) as described in the analysis of galactosyloligosaccharides in Yakult products (Kimura et al. 1995) and commercial cows' milk (Kimura et al. 1997). Each sample was dried in a screw-cap tube $(16 \times 100 \mathrm{~mm})$ under reduced pressure at room temperature. Coupling reagent $(100 \mu \mathrm{l})(1.1 \mathrm{~g}$ 2-aminopyridine (Wako Pure Chemical Industries Ltd., Osaka, Japan) in $650 \mu \mathrm{l}$ acetic acid) was added to the 
residue in the tube. The tube was sealed with a screw cap and heated at $90^{\circ} \mathrm{C}$ for $1 \mathrm{~h}$, and then $100 \mu \mathrm{l}$ of freshly prepared reducing reagent (195 $\mathrm{mg}$ borane-dimethylamine complex (Aldrich Chemical Company Inc., USA) in $1 \mathrm{ml}$ acetic acid) was added. The tube was sealed again and heated at $80^{\circ} \mathrm{C}$ for $50 \mathrm{~min}$. The resulting pyridylaminated derivatives were separated from the reaction mixture by electrodialysis using a Microacilyzer model G-1 (Asahi Kasei Co., Kanagawa, Japan) with a 120-10 cartridge, followed by freeze-drying. The detail of the electrodialysis procedure has been described elsewhere by Kimura et al. (1995).

Pyridylaminated oligosaccharide fractions were subjected to reverse-phase HPLC. The analysis was performed with an Inertsil ODS-3V column $(4.6 \times 250 \mathrm{~mm}$, packed with $5 \mu \mathrm{l}$ particles having $10 \mathrm{~nm}$ pore size; GL Sciences Inc., Tokyo, Japan) at $25^{\circ} \mathrm{C}$ in a column oven (CO-8020; Tosoh Co., Tokyo, Japan). The eluate was controlled by a multi-pump (CCMP-II; Tosoh) equipped with a CCP controller (PX-8020; Tosoh). Ammonium acetate (a/M pH 5.5) was used as solution $A$ and $5 \%(\mathrm{v} / \mathrm{v})$ methanol in $0 \cdot 1 \mathrm{M}$-ammonium acetate solution $(\mathrm{pH} 5.5)$ as solution B. Elution was done at a flow rate of $1 \mathrm{ml} / \mathrm{min}$ and with a linear gradient from 0 to $100 \%$ of solution $\mathrm{B}$ in 60 min. Detection was done with a MCPD-3600 Photodiode Array Detector (Otsuka Electronics Co. Ltd,
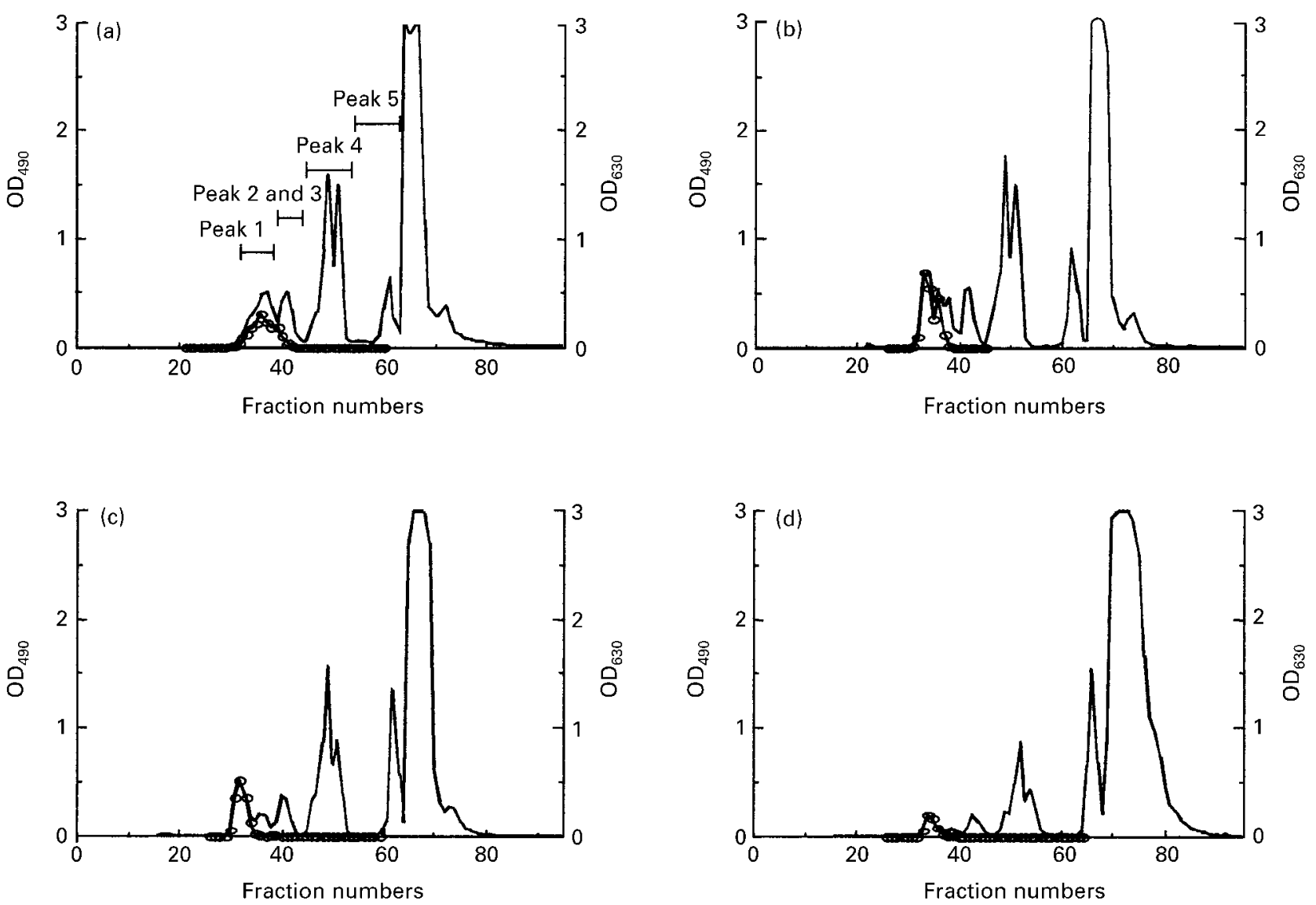

Fig. 1. Gel chromatograms of the carbohydrate fractions separated on a Bio Gel P-2 $(2.6 \times 100 \mathrm{~cm}) \mathrm{column}$. Each fraction was analysed for hexose using the phenol- $\mathrm{H}_{2} \mathrm{SO}_{4}$ method at $490 \mathrm{~nm}(-)$ and for sialic acid with the periodate-resorcinol method at $630 \mathrm{~nm}(-\mathrm{O}-)$. $\mathrm{OD}_{490}$, optical density at $490 \mathrm{~nm}$; $\mathrm{OD}_{630}$, optical density at $630 \mathrm{~nm}$. The carbohydrate fractions were separated from human milk at (a) 4, (b) 10, (c) 30 and (d) $100 \mathrm{~d}$ post partum. 
Table 1. The neutral oligosaccharides quantified in human milk at several stages of lactation*

\begin{tabular}{|c|c|}
\hline Oligosaccharide & Structure \\
\hline $\begin{array}{l}\text { 3-FL } \\
2^{\prime}-\text {-FL } \\
\text { LNDFT } \\
\text { LNT } \\
\text { LNnT } \\
\text { LNFP I } \\
\text { LNFP II } \\
\text { LNFP III } \\
\text { LNDFH I } \\
\text { LNDFH II } \\
\text { LNH } \\
\text { LNnH }\end{array}$ & 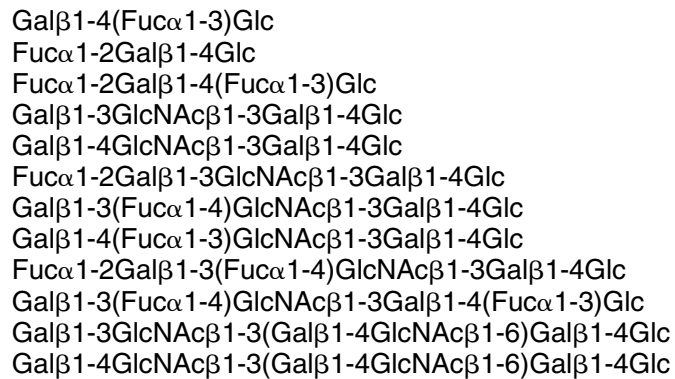 \\
\hline
\end{tabular}

3-FL, 3-fucosyllactose; 2'-FL, 2'-fucosyllactose, LNDFT, lacto-N-difucotetraose, LNT, lacto-N-tetraose, LNnT, lacto-N-neotetraose, LNFP, lacto-N-fucopentaose; LNDFH, lacto-N-difucohexaose; LNH, lacto-N-hexaose; LNnH, lacto-N-neohexaose; Gal, galactose, Fuc, fucose; Glc, glucose; Ac, acetyl.

${ }^{\star}$ For details of procedures, see p. 62.

by anion exchange chromatography and Bio Gel P-4 column chromatography, and designated as peak 3 .

The area of peak 3, which contained the higher oligosaccharides, decreased relative to the others from 4 to $10 \mathrm{~d}$ postpartum. Subsequently, there was little change up to $100 \mathrm{~d}$ postpartum. The area of peak 4 , which contained tetra- to hexa-saccharides, increased relative to the other peaks from 4 to $10 \mathrm{~d}$ postpartum, and then decreased up to $100 \mathrm{~d}$ postpartum. On the other hand, the area of peak 5 , which contained tri- to tetra-saccharides, increased up to $100 \mathrm{~d}$ postpartum.

The oligosaccharides quantified in the present study are listed in Table 1 . In recovery studies, greater than $90 \%$ of each pyridylaminated oligosaccharide was recovered. It was shown that each peak contained the following oligosaccharides by HPLC of peak 3 to 5 : LNH and LNnH in peak 3; LNT, LNnT, LNFP I, LNFP II, LNFP III, LNDFH I and LNDFH II in peak 4; 3-FL, $2^{\prime}$-FL and LNDFT in peak 5 (Fig. 2) LNFP II and LNDFH II in peak 4 , and $2^{\prime}$-FL and LNDFT in peak 5 were not separated from each other by the HPLC. Because peak 3 had too many peaks derived from higher oligosaccharides, we could not quantify LNH and LNnH.

The quantitative value of each and the mean concentration of each oligosaccharide in each lactation period are shown in Table 2. Characteristic changes in each oligosaccharide can be seen. The total oligosaccharide concentration calculated by summing all the compounds in the

Table 2. Concentration of each oligosaccharide (mg/l) in human milk at $4,10,30$ and $100 \mathrm{~d}$ postpartum* (Mean values with their standard errors, and ranges)

\begin{tabular}{|c|c|c|c|c|c|c|c|c|}
\hline & \multicolumn{8}{|c|}{ Day of lactation } \\
\hline & \multicolumn{2}{|c|}{$4(n 16)$} & \multicolumn{2}{|c|}{$10(n 15)$} & \multicolumn{2}{|c|}{$30(n 16)$} & \multicolumn{2}{|c|}{$100(n 15)$} \\
\hline & Mean & SE & Mean & SE & Mean & SE & Mean & SE \\
\hline 3-FL & 232.5 & $68 \cdot 1$ & 280.9 & $55 \cdot 3$ & 427.5 & $105 \cdot 1$ & $452 \cdot 0$ & 127.5 \\
\hline Range & \multicolumn{2}{|c|}{$13 \cdot 8-1009 \cdot 3$} & \multicolumn{2}{|c|}{$3.7-766 \cdot 0$} & \multicolumn{2}{|c|}{$23 \cdot 0-1423 \cdot 6$} & \multicolumn{2}{|c|}{$0.0-1491.8$} \\
\hline $2^{\prime}-\mathrm{FL}+\mathrm{LNDFT}$ & 403.5 & $199 \cdot 8$ & $685 \cdot 5$ & $265 \cdot 0$ & $580 \cdot 6$ & $209 \cdot 4$ & 89.4 & $39 \cdot 3$ \\
\hline Range & \multicolumn{2}{|c|}{$0.0-2482 \cdot 5$} & \multicolumn{2}{|c|}{$0.0-3448.2$} & \multicolumn{2}{|c|}{$0 \cdot 0-2448 \cdot 7$} & \multicolumn{2}{|c|}{$0.0-599.1$} \\
\hline LNT & $347 \cdot 2$ & $79 \cdot 1$ & $360 \cdot 9$ & 78.5 & $259 \cdot 6$ & $45 \cdot 6$ & $96 \cdot 1$ & $40 \cdot 4$ \\
\hline Range & \multicolumn{2}{|c|}{$8 \cdot 7-988 \cdot 7$} & \multicolumn{2}{|c|}{$11 \cdot 6-830 \cdot 2$} & \multicolumn{2}{|c|}{$6 \cdot 7-565 \cdot 4$} & \multicolumn{2}{|c|}{$2 \cdot 9-648 \cdot 2$} \\
\hline LNnT & 211.4 & $42 \cdot 0$ & $208 \cdot 8$ & $51 \cdot 3$ & 155.5 & $58 \cdot 3$ & 44.4 & $13 \cdot 0$ \\
\hline Range & \multicolumn{2}{|c|}{$10 \cdot 6-516 \cdot 7$} & \multicolumn{2}{|c|}{$0.0-718.2$} & \multicolumn{2}{|c|}{$0.0-768.7$} & \multicolumn{2}{|c|}{$0.0-146 \cdot 0$} \\
\hline LNFP I & 968.7 & $205 \cdot 6$ & 1137.5 & 319.9 & $742 \cdot 7$ & 204.7 & $268 \cdot 1$ & $109 \cdot 1$ \\
\hline Range & \multicolumn{2}{|c|}{$62 \cdot 1-2772 \cdot 5$} & \multicolumn{2}{|c|}{$0.0-4168.4$} & \multicolumn{2}{|c|}{$9.1-2665 \cdot 3$} & \multicolumn{2}{|c|}{$0.0-1770.6$} \\
\hline LNFP III & $52 \cdot 3$ & 8.9 & $71 \cdot 6$ & $17 \cdot 7$ & 73.5 & $16 \cdot 2$ & 71.9 & $22 \cdot 0$ \\
\hline Range & \multicolumn{2}{|c|}{$4 \cdot 7-108 \cdot 4$} & \multicolumn{2}{|c|}{$4 \cdot 3-217 \cdot 1$} & \multicolumn{2}{|c|}{$4 \cdot 7-217 \cdot 6$} & \multicolumn{2}{|c|}{$8 \cdot 3-296.9$} \\
\hline LNFP II + LNDFH II & 323.4 & 88.8 & 457.9 & $143 \cdot 0$ & 557.8 & $150 \cdot 1$ & $301 \cdot 7$ & $86 \cdot 8$ \\
\hline Range & \multicolumn{2}{|c|}{$17 \cdot 5-1057 \cdot 5$} & \multicolumn{2}{|c|}{$21 \cdot 2-2292 \cdot 0$} & \multicolumn{2}{|c|}{$33 \cdot 8-2238 \cdot 0$} & \multicolumn{2}{|c|}{$27 \cdot 1-1095 \cdot 3$} \\
\hline LNDFH I & $144 \cdot 6$ & 29.9 & $225 \cdot 6$ & $62 \cdot 3$ & $156 \cdot 5$ & $37 \cdot 0$ & $90 \cdot 4$ & $25 \cdot 3$ \\
\hline Range & \multicolumn{2}{|c|}{$0.0-343.8$} & \multicolumn{2}{|c|}{$0.0-695.3$} & $2 \cdot 7$ & $36 \cdot 3$ & $0 \cdot 0-$ & $4 \cdot 8$ \\
\hline Total† & & & 34 & & & & & \\
\hline
\end{tabular}

3-FL, 3-fucosyllactose; 2'-FL, 2'-fucosyllactose; LNDFT, lacto-N-difucotetraose, LNT, lacto-N-tetraose, LNnT, lacto-N-neotetraose, LNFP, lacto-N-fucopentaose; LNDFH, lacto-N-difucohexaose.

* For details of procedures see p. 62.

†The sum of the above oligosaccharides quantified in human milk in the present study. 

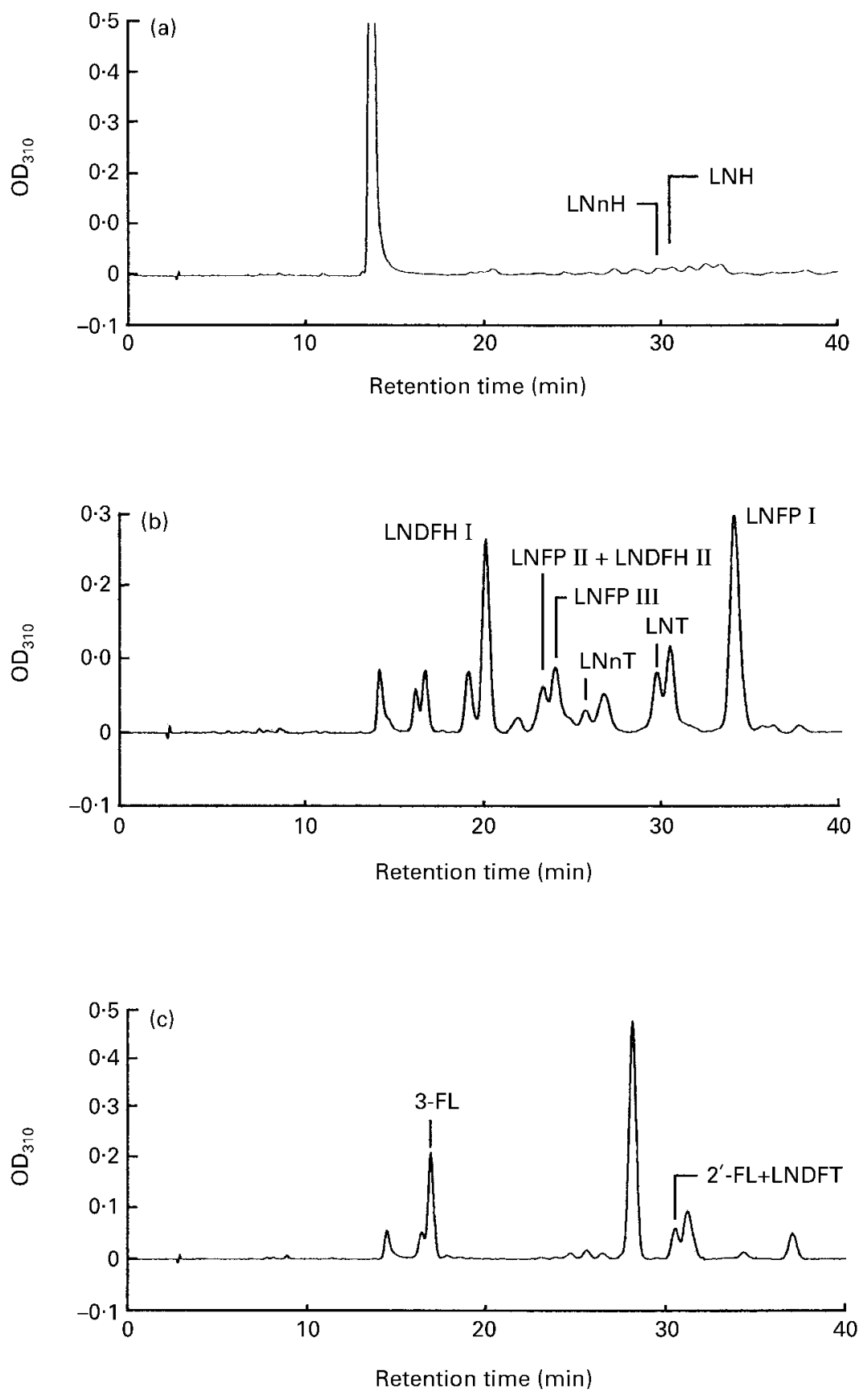

Fig. 2. High-performance liquid chromatogram of the derivatives with pyridylamination of (a), peak 3; (b), peak 4; (c), peak 5 separated from human milk at $4 \mathrm{~d}$ postpartum. $\mathrm{OD}_{310}$, optical density at $310 \mathrm{~nm}$; $\mathrm{LNnH}$, lacto-N-neohexaose; $\mathrm{LNH}$, lacto-N-hexaose; LNDFH, lacto-N-difucohexaose; LNFP, lacto-N-fucopentaose; LNT, lacto-N-tetraose; LNnT, lacto-N-neotetraose; 3-FL, 3-fucosyllactose; 2'-FL, 2'-fucosyllactose; LNDFT, lacto-N-difucotetraose.

present study increased from $2683.6 \mathrm{mg} / \mathrm{l}$ at $4 \mathrm{~d}$ postpartum to $3426.8 \mathrm{mg} / \mathrm{l}$ at $10 \mathrm{~d}$ postpartum, and then decreased to $1414.0 \mathrm{mg} / \mathrm{l}$ at $100 \mathrm{~d}$ postpartum. However, the concentration of individual oligosaccharides varied greatly among the donors and some oligosaccharides were not detected in specific lactation periods of some donors.

No relationship was observed between oligosaccharide content and the $\mathrm{ABO}$ blood group of the donors in the present study.

\section{Discussion}

These findings provide precise information about the concentration and pattern of change of individual neutral oligosaccharides in human milk. Large differences were observed between the data obtained in the present study and those reported by Coppa et al. (1999) for Italian women (Fig. 3). Coppa et al. (1999) may have overestimated the amounts because of overlapping of the 

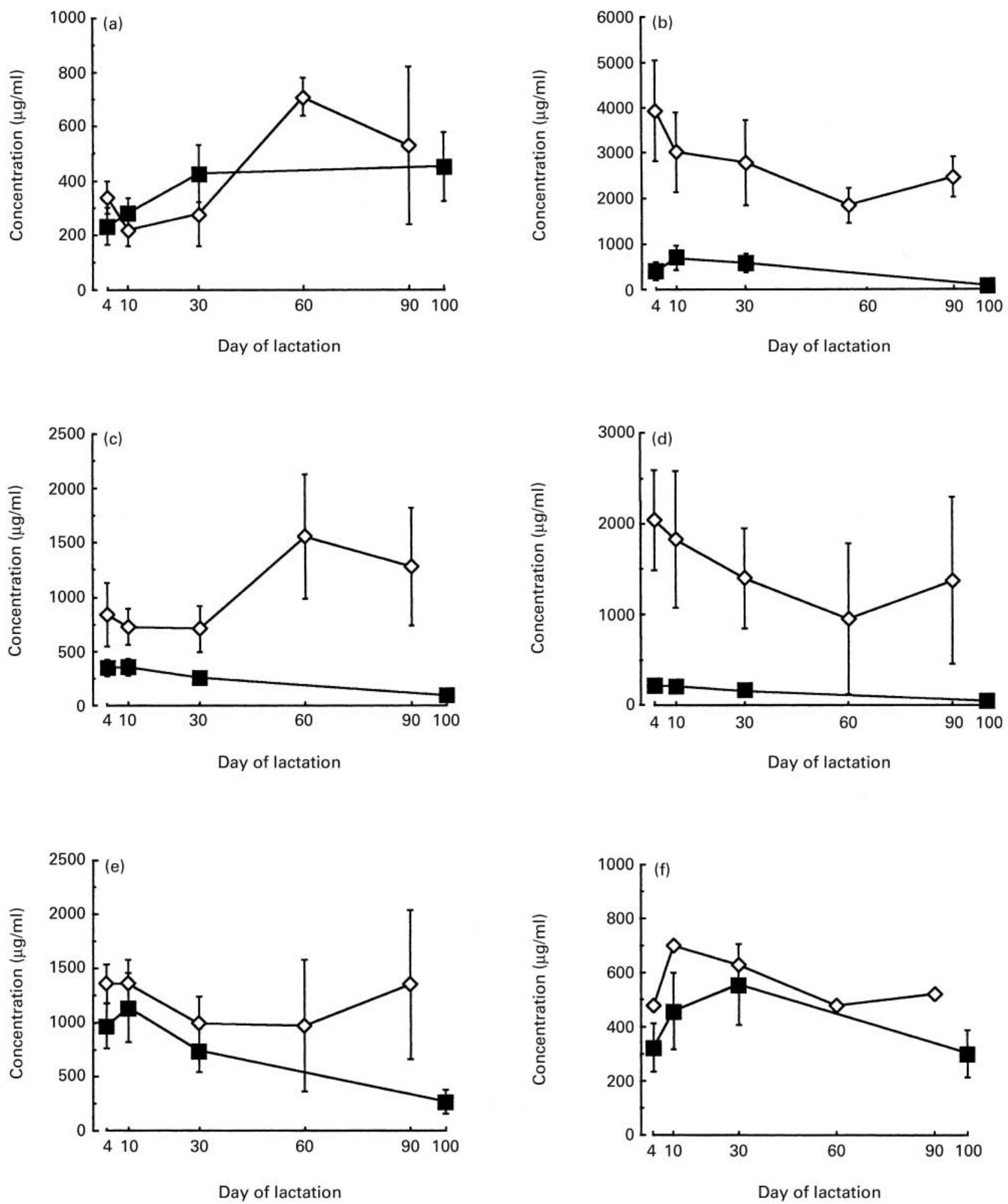

Fig. 3. Changes in concentration of major oligosaccharides in human milk during the course of lactation. ( $\square$ ), Data from present study

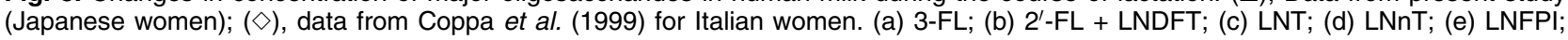
(f) LNFPII + LNDFHII.

peaks corresponding to several oligosaccharides. However, the differences could also be due to the small sample sizes in both studies and the natural variability among women. It is well known that the oligosaccharide content of milk varies according to blood groups Lewis $\mathrm{a}, \mathrm{b}$, or $\mathrm{x}$ and their different secretory status (Thurl et al. 1997). Oligosaccharide content in milk has also been suggested to vary according to racial group as shown by Erney et al. (2000). Methodological issues may also explain the differences between the two studies. We used HPLC analysis 
after pyridylamination of the fractions separated by gel filtration of the milk carbohydrates, whereas Coppa et al. (1999) used high $\mathrm{pH}$ anion exchange chromatography of the fractions separated directly from the milk by centrifugation and filtration with a $0.22 \mu \mathrm{m}$ membrane. At the present time, it is not known whether one method is more accurate than the other.

In particular, the core oligosaccharides such as LNT, LNnT, LNH and LNnH in the present study were significantly lower than those reported by Coppa et al. (1999). It is possible that there is lower activity of the enzymes $\beta 3$ or $\beta 6 \mathrm{~N}$-acetylglucosaminyltransferase, which are involved in the biosynthesis of those oligosaccharides, in the lactating mammary glands of Japanese women.

In the present study, the contents of $\alpha$ 1-2 fucosyloligosaccharides increased from 4 to $10 \mathrm{~d}$ postpartum, and that of $\alpha$ 1-3/4 fucosyloligosaccharides increased from 4 to $30 \mathrm{~d}$ postpartum. This suggests that $\alpha 1-2$ and $\alpha 1-3 / 4$ fucosyltransferase activities are high in early lactation. The content of $\alpha$ 1-2 fucosyloligosaccharides started to decrease from $10 \mathrm{~d}$ postpartum, suggesting that $\alpha 1-2$ fucosyltransferase activity decreased at this stage. On the other hand, the contents of $\alpha 1-3 / 4$ fucosyloligosaccharides decreased from 30 to $100 \mathrm{~d}$ postpartum, suggesting that $\alpha$ 1-3/4 fucosyltransferase activity decreased from 30 to $100 \mathrm{~d}$ postpartum. These data also suggest that total fucosyltransferase activity is high in early lactation and decreases from 30 to $100 \mathrm{~d}$ postpartum. Wiederschain \& Newburg (1995) reported that fucosyltransferase activity in milk is maximal in colostrum, and then decreases temporarily, followed by an increase until $30 \mathrm{~d}$ postpartum. In the study of Coppa et al. (1999) the contents of $2^{\prime}$-FL + LNDFT and LNFP I were maximal at $4 \mathrm{~d}$ postpartum, whereas the content of LNFP II + LNDFH II was maximal at $10 \mathrm{~d}$ postpartum. The content of 3-FL was maximal at $60 \mathrm{~d}$ postpartum and then decreased. The difference in milk fucosyloligosaccharides contents between our data and Coppa et al. (1999) may be caused by differences in fucosyltransferase activities in the lactating mammary glands of Japanese $v$. Italian women.

Recently, Chaturvedi et al. (2001) determined the level of individual oligosaccharides in the milk of Mexican women. The $\alpha 1-3 / 4$ fucosyloligosaccharide contents were similar to those of our study, whereas $\alpha$ 1-2 fucosyloligosaccharide content was higher, increasing from 7 to 14 weeks postpartum. Again, we do not know whether these differences are determined by ethnicity, Lewis blood group status, methodology or the small numbers of mothers. Only large-scale studies of lactating women in different populations using uniform methods will answer these questions.

Although we determined the content of the representative neutral oligosaccharides, human milk contains more than 100 different oligosaccharides (Newburg \& Neubauer, 1995). Many oligosaccharides have large molecular weights. Unfortunately at present we cannot determine the content of individual higher oligosaccharides with any technique. The difference between the combined total of the oligosaccharides determined in the present study and the total oligosaccharide content of human milk reported in the literature $(12-14 \mathrm{~g} / \mathrm{l}$ in mature milk and $20-24 \mathrm{~g} / 1$ in colostrum) reflects the large quantities of acidic and large-molecular-weight oligosaccharides in human milk.

It has been postulated that some of the human milk oligosaccharides act as analogues or homologues to host cell surface receptors for pathogens. LNT or LNnT inhibit attachment of Streptococcous pneumoniae (Andersson et al. 1986) to pharyngeal or buccal epithelial cells, and fucosyloligosaccharides inhibit the adhesion of enteropathogenic Escherichia coli (Cravioto et al. 1991; Cervantes et al. 1995) or heat-stable enterotoxin of E. coli (Cervantes et al. 1995; Newburg et al. 1999) to intestinal cells. Our data, showing that neutral oligosaccharide content is very high in colostrum and gradually declines as lactation proceeds, supports the hypothesis that oligosaccharides function as anti-infective factors in newborn infants whose immune systems are not mature.

Many of the milk oligosaccharides are growth factors for the intestinal micro-organisms Bifidobacterium, among the most concentrated being LNT and LNFP I (Gÿorgy \& Rose, 1955). The high content of oligosaccharides in colostrum would be favourable for establishment of the Bifidus flora in the newborn colon. The oligosaccharide concentrations in the colostrums of preterm milk have been found to be significantly higher than full-term colostrum (Coppa et al. 1997), suggesting that the oligosaccharides act as anti-pathogenic factors and Bifidobacterium growth factors for the preterm babies, too.

Lactating mothers produce about $600-900 \mathrm{ml} \mathrm{milk/d}$ through the first year after delivery (Neville, 1995), and their babies take a few grams of oligosaccharides/d. On the other hand, the content of oligosaccharides in infant formula is low because bovine milk, the raw material for infant formula, has a significantly lower oligosaccharides content than human milk (Neeser et al. 1991). Since the composition of infant formulas is modelled on that of human milk, consideration should be given to the addition of human milk oligosaccharides, the third largest solute in human milk, to infant formula. Attempts to synthesise LNT, LNnT (Murata et al. 1999a) and fucosyloligosaccharides (Murata et al. 1999b; Farkas et al. 2000) have been made by transglycosylation with glycosyltransferase or glycosidase specifically for the purpose of addition to infant formula. Our data provide a basis for estimating the amounts that might be considered appropriate.

\section{References}

Andersson B, Porras O, Hanson LÅ, Lagergård T \& SvanborgEdén C (1986) Inhibition of attachment of Streptococcus pneumoniae and Haemophilus influenzae by human milk and receptor oligosaccharides. Journal of Infectious Disease 153, $232-237$.

Cervantes LE, Newburg DS \& Ruiz-Palacios GM (1995) \& 1-2 fucosylated chains (H-2 and Lewis b) are the main human milk receptor analogs for Campylobacter. Pediatric Research 37, 171 A.

Chaturvedi P, Warren CD, Altaye M, Morrow AL, Ruiz-Palacios G, Pickering LK \& Newburg DS (2001) Fucosylated human milk oligosaccharides vary between individuals and over the course of lactation. Glycobiology 11, 365-372. 
Chaturvedi P, Warren CD, Ruiz-Palacios GM, Pickering LK \& Newburg DS (1997) Milk oligosaccharide profiles by reversed-phase HPLC of their perbenzoylated derivatives. Analytical Biochemistry 251, 89-97.

Coppa GV, Bruni S, Zampini L, Galeazzi T, Capretti R, Facinelli B \& Gabrielli O (2000) Human milk oligosaccharides inhibit the adhesion of Listeria monocytogenes, Escherichia coli, Vibrio cholerae and Salmonella fyris. In In 20th International Carbohydrate Symposium, Hamburg, 27 August-1 September 2000. Abstract, International Carbohydrate Organization.

Coppa GV, Gabrielli O, Giorge P, Catassi C, Montanari MP, Varaldo PE \& Nichols BL (1990) Preliminary study of breastfeeding and bacterial adhesion to uroepithelial cells. Lancet 335, 569-571.

Coppa GV, Pierani P, Zampini L, Carloni I \& Gabrielli O (1999) Oligosaccharides in human milk during different phases of lactation. Acta Paediatrica 430, Suppl., 89-94.

Coppa GV, Pierani P, Zampini L, Gabrielli O, Carlucci A, Catassi C \& Giorgi PL (1997) Lactose, oligosaccharide and monosaccharide content of milk from mothers delivering preterm newborns over the first month of lactation. Minerva Pediatrica 49, 471-475.

Cravioto A, Tello A, Villafán H, Ruiz J, Vedovo S \& Nesser JR (1991) Inhibition of localized adhesion of enteropathogenic Escheichia coli to Hep-2 cells by immunoglobulin and oligosaccharide fractions of human colostrums and breast milk. Journal of Infectious Disease 163, 1247-1255.

Dai D, Nanthkumar NN, Newburg DS \& Walker WA (1990) Role of oligosaccharides and glycoconjugates in intestinal host defense. Journal of Pediatric Gastroenterology and Nutrition 30, s23-s33.

Dubois M, Gilles KA, Hamilton JK, Rebers PA \& Smith F (1956) Colorimetric method for determination of sugars and related substances. Analytical Chemistry 28, 350-356.

Erney RM, Malone WT, Skelding MB, Marcon AA, KlemanLeyer KM, O'Ryan ML, Ruiz-Palacios G, Hilty MD, Pickering LK \& Prieto PA (2000) Variability of human milk neutral oligosaccharides in a diverse population. Journal of Pediatric Gastroenterology and Nutrition 30, 181-192.

Farkas E, Thiem J \& Ajisaka K (2000) Enzymatic synthesis of fucose-containing disaccharides employing the partially purified $\alpha$-L-fucosidase from Penicillium multicolor. Carbohydrate Research 328, 293-299.

Gÿorgy P, Norris RF \& Rose CS (1954) Bifidus factor. I. A variant of Lactobacillus bifidus requiring a special growth factor. Archives of Biochemistry and Biophysics 48, 193-201.

Gÿorgy P \& Rose CS (1955) Microbiological Studies on Growth Factor for L. bifidus var. pennsylvanicus. Proceedings of the Society for Experimental Biology and Medicine 90, 219-223.

Hase S (1994) High-performance liquid chromatography of pyridylaminated saccharides. Methods in Enzymology 230, $225-237$.

Idota T, Kawakami H, Murakami Y \& Sugawara M (1995) Inhibition of cholera toxin by human milk fractions and sialyllactose. Bioscience, Biotechnology, and Biochemistry 59, 417-419.

Jourdian GW, Dean L \& Roseman S (1971) The sialic acids XI. A periodate-resorcinol method for quantitative estimation of free sialic acids and their glycosides. Journal of Biological Chemistry 246, 430-435.

Kimura K, Matsumoto K, Ishihara C, Harada K \& Miyagi A (1995) Structure determination of galacto-oligosaccharides by pyridylamintion and NMR spectroscopy. Carbohydrate Research 270, 33-42.

Kimura K, Watanabe Y, Matsumoto K \& Miyagi A (1997) Studies on the natural galacto-oligosaccharides in commercial cow's milk. YAKULT Institute Report 17, 1-7.
Kondo A, Suzuki J, Kuraya N, Hase S, Kato I \& Ikenaka T (1990) Improved method for fluorescence labeling of sugar chains with sialic acid residues. Agricultural and Biological Chemistry 54, 2169-2170.

Kunz C \& Rudloff S (1993) Biological functions of oligosaccharides in human milk. Acta Paediatrica 82, 903-912.

McVeagh P \& Miller JB (1997) Human milk oligosaccharides: only the breast. Journal of Paediatrics and Child Health 33, 281-286.

Miller JB \& McVeagh P (1999) Human milk oligosaccharides: 130 reasons to breast-feed. British Journal of Nutrition 82, 333-335.

Murata T, Inukai T, Suzuki M, Yamagishi M \& Usui T (1999a) Facile enzymatic conversion of lactose into lacto-N-tetraose and lacto-N-neotetraose. Glycoconjugate Journal 16, 189-195.

Murata T, Morimoto S, Zeng X, Watanabe S \& Usui T (1999b) Enzymatic synthesis of $\alpha$-L-fucosyl-N-acetyllactosamines and $3^{\prime}-\mathrm{O}-\alpha$-L-fucosyllactose utilizing $\alpha$-L-fucosidases. Carbohydrate Research 320, 192-199.

Nakhla T, Fu D, Zoph D, Brodsky NL \& Hurt H (1999) Neutral oligosaccharide content of preterm human milk. British Journal of Nutrition 82, 361-367.

Neeser JR, Golliard M \& Vedovo SD (1991) Quantitative determination of complex carbohydrates in bovine milk and in milk-based infant formulas. Journal of Dairy Science 74, 2860-2871.

Neville MC (1995) Volume of Caloric Density of Human Milk. In Handbook of Milk Composition, pp. 99-113 [RG Jensen, editor]. San Diego, CA: Academic Press.

Newburg DS (1996) Oligosaccharides and glycoconjugates in human milk: Their role in host defense. Journal of Mammary Gland Biology and Neoplasia 1, 271-283.

Newburg DS (1997) Do the binding properties of oligosaccharides in milk protect human infants from gastrointestinal bacteria? Journal of Nutrition 127, Suppl. 5, 980s-984s.

Newburg DS (1999) Human milk glycoconjugates that inhibit pathogens. Current Medicinal Chemistry 6, 117-127.

Newburg DS (2000) Oligosaccharides in human milk and bacterial colonization. Journal of Pediatric Gastroenterology and Nutrition 30, s8-s17.

Newburg DS \& Neubauer SH (1995) Carbohydrates in milks: Analysis, Quantities, and Significance. In Handbook of Milk Composition, pp. 273-349 [RG Jensen, editor]. San Diego, CA: Academic Press

Newburg DS, Pickering LK, McCluer RH \& Cleary TG (1999) Fucosylated oligosaccharides of human milk protect suckling mice from heat-stable enterotoxin of Eschericiha coli. Journal of Infectious Disease 162, 1075-1080.

Portelli J, Gordon A \& May JT (1998) Effect of human milk sialyllactose on Cytomegalovirus (letter). European Journal of Clinical Microbiology and Infectious Diseases 17, 66.

Saito T, Tsuji T, Kitazawa H, Kawai Y \& Itoh T (1998) Application of two-dimensional mapping for an Analysis of Galactosyllactoses in yogurt. Bioscience, Biotechnology, and Biochemistry 62, 1445-1447.

Stahl B, Thurl S, Zeng J, Karas M, Hillenkamp F, Steup M \& Sawatzki G (1994) Oligosaccharides from human milk as revealed by matrix-assisted laser desorption/ionization mass spectrometry. Analytical Biochemistry 223, 218-226.

Suzuki J, Kondo A, Kato I, Hase S \& Ikenaka T (1991) Analysis by high-performance anion-exchange chromatography of component sugar as their fluorescent pyridylamino derivatives. Agricultural and Biological Chemistry 55, 283-284.

Takemoto H, Hase S \& Ikenaka T (1985) Microquantitative analysis of neutral and amino sugars as fluorescent pyridylamino derivatives by high-performance liquid chromatography. Analytical Biochemistry 145, 245-250. 
Thurl S, Henker J, Siegel M, Tovar K \& Sawatzki G (1997) Detection of four human milk groups with respect to Lewis blood group dependent oligosaccharides. Glycoconjugate Journal 14, 795-799.

Watkins WM (1996) Glycoproteins: Their Composition, Structure and Function, pp. 462-512 [A Gottschalk, editor]. Amsterdam: Elsevier.

Wiederschain GY \& Newburg DS (1995) Human milk Fucosyltransferase and $\alpha$-L-fucosidase activities change during the course of lactation. Journal of Nutritional Biochemistry 6, 582-587.

Yanagida K, Natsuka S \& Hase S (1999) A pyridylamination method aimed at automatic oligosaccharide analysis of $\mathrm{N}$-linked sugar chains. Analytical Biochemistry 274, 229-234. Yasuno S, Kokubo K \& Kamei M (1999) New method for determining the sugar composition of glycoproteins, glycolipids, and oligosaccharides by high-performance liquid chromatography. Bioscience, Biotechnology, and Biochemistry 63, $1353-1359$.

Yoshioka H, Iseki K \& Fujita K (1983) Development and differences of intestinal flora in the neonatal period in breast-fed and bottle-fed infants. Pediatrics 72, 317-321.

Zopf D \& Roth S (1996) Oligosaccharide anti-infective agents. Lancet 347, 1017-1021. 